\title{
On the performance of MIMO RFID backscattering channels
}

\author{
Chen $\mathrm{He}^{1 *}$, Xun Chen ${ }^{1}$, Zhen Jane Wang ${ }^{1}$ and Weifeng $\mathrm{Su}^{2}$
}

\begin{abstract}
In order to increase the reliability of data transmission, using multiple antennas in radio frequency identification (RFID) systems has been investigated by researchers, mainly through measurements and simulations. The multiple-input multiple-output (MIMO) RFID backscattering channel exhibits a special type of cascaded structure rather than that of other better-studied cascaded channels such as the keyhole fading and the double scattering fading. In this article, we analytically study the bit error rate performances of the MIMO RFID channel under two transmission schemes, the identical signaling transmission scheme and the orthogonal space-time coding scheme (OSTBC). We show that the diversity order of the MIMO RFID channel is $\min (N, L)$ under the identical transmission scheme, and the diversity order is $L$ under the OSTBC scheme, where $L$ is the number of tag antennas and $N$ is the number of reader receiving antennas. A performance bottleneck is also observed in the MIMO RFID channel. Our results can provide useful guidance on designing an RFID system with multiple antennas.
\end{abstract}

Keywords: MIMO systems, RFID, Backscattering channel, Cascaded structures, STBC, Diversity gain

\section{Introduction}

Radio frequency identification (RFID) is a wireless communication technology that allows an object to be identified remotely, which has many applications including inventory checking, access control, transport payment, electronic vehicle registration, product tracking, and secure automobile keys [1]. A typical RFID system includes major components such as readers (also known as interrogators) and tags (also known as labels), as well as RFID software or RFID middleware [2]. An RFID tag is a small electronic device which has a unique ID. The tags can be categorized into active and passive tags. An active tag has an RF transmitter and utilizes its internal battery to continuously power its RF communication circuitry, while a passive tag does not have an RF transmitter and it modulates a carrier signal received from an interrogator by its antenna load impedance. Usually, a passive tag does not have its own battery and powers its circuitry by using the carrier signal energy, but it can be battery assisted. For passive RF tags, the range increase caused by multiple RF tag antennas will be limited by the RF tag chip

\footnotetext{
*Correspondence: chenh@ece.ubc.ca

1 Department of Electrical and Computer Engineering, University of British Columbia, Vancouver, Canada

Full list of author information is available at the end of the article
}

sensitivity [3]. The sensitivity is strongly dependent upon the design of the tag's RF circuitry [4]. During the last couples of years, it was shown that the improved circuitry design $[3,5,6]$ can decrease this limitation.

Most RFID applications deployed today use passive tags because they usually do not require internal batteries and have longer life expectancy. Measurements in $[7,8]$ showed that the RFID channel in passive systems could be modeled as a cascaded channel with a forward channel and a backscattering channel, and both the sub-channels are Rayleigh distributed in rich scattering environments [9]. This cascaded channel fades deeper than the Rayleigh channel and hence can reduce the data transmission reliability.

To allow reliable data transmission, researchers [7,9-11] investigated multiple-input multiple-output (MIMO) settings in the RFID backscattering channel and both bit error rate (BER) and reading range improvements were observed. The advantages of the MIMO RFID technology over the single-antenna RFID allow many potential applications in the areas of accurate tracking, identifications, since it can significantly increase the reliability and throughput. For instance, in [12], a tracking problem was investigated for RF tags with multiple antennas and experimental results showed improved accuracy. To achieve

\section{Springer}

(c) 2012 He et al: licensee Springer. This is an Open Access article distributed under the terms of the Creative Commons Attribution License (http://creativecommons.org/licenses/by/2.0), which permits unrestricted use, distribution, and reproduction in any medium, provided the original work is properly cited. 
good performance, a sufficient separation between tag antennas is needed and hence a very high-frequency band is required for operations of RF tags with multiple antennas due to the small size of an RF tag. Fortunately, the unlicensed frequency band $5.8 \mathrm{GHz}$ is available for backscatter radio applications. This above ultra-high frequency band has several potential advantages for backscatter radio systems such as increased antenna gain, reduced object attachment losses [13]. It has already been used in a passive backscatter radio system for monitoring [14]. To the best of the authors' knowledge, the current studies about the performances of the MIMO RFID channel were mostly based on the measurements and/or Monte Carlo simulations. The main purpose of this study is to provide a fundamental, analytical study of the behavior of the MIMO RFID channel, and to provide useful guidances on the design of potential RFID systems with multiple antennas.

\section{Channel model}

\section{Passive tag signaling model}

In a passive RFID system, a tag antenna simply reflects the unmodulated wave sent from the reader transmitter, as illustrated in Figure 1. The ID information of the RF tag depends on the reflection coefficient $\Delta(t)$ of the tag antenna load. The general $M \times L \times N$ dyadic backscattering channel for representing a MIMO passive channel was first described in [9]. This channel consists of $M$ reader transmitter antennas (from which a query is sent and the energy is transmitted), $L \mathrm{RF}$ tag antennas, and $N$ reader receiver antennas. The baseband representation of the received signals at time $t$ can be expressed in a matrix form as [9]

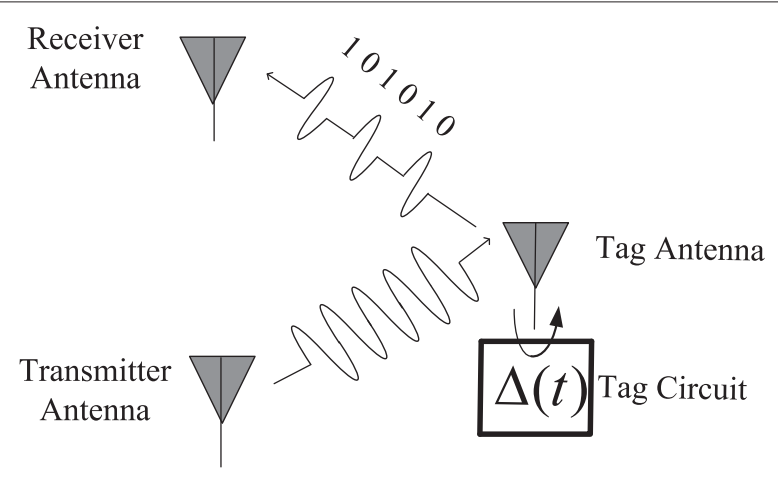

Figure 1 To read a passive RF tag, the RF reader transmitter broadcasts an unmodulated carrier signal, and then the RF tag conveys its information (e.g., the ID of the tag) to the reader by reflecting the unmodulated carrier signal back to the reader receiver using load modulation. The reflection coefficient $\Delta(t)$ of the tag antenna load, which actually represents the tag information, can be changed by switching the RF tag antenna load between different states [9].

$$
\begin{aligned}
\mathbf{r}(t)= & \frac{1}{2} \int_{-\infty}^{\infty} \int_{-\infty}^{\infty} \mathbf{H}^{b}\left(\tau_{b} ; t\right) \mathbf{S}(t) \mathbf{H}^{f}\left(\tau_{f} ; t\right) \mathbf{x}\left(t-\tau_{b}-\tau_{f}\right) \\
& \times d \tau_{b} d \tau_{f}+\mathbf{w}(t),
\end{aligned}
$$

where the $M \times 1$ vector $\mathbf{x}(t)$ represents unmodulated waves (carrier signals) sent out from the reader transmitters, $\mathbf{r}(t)$ is an $N \times 1$ vector of received signals at the reader receivers, and the $N \times 1$ vector $\mathbf{w}(t)$ represents corresponding noises at the reader receivers. The matrices $\mathbf{H}^{f}\left(\tau_{f} ; t\right)$ of size $L \times M$ and $\mathbf{H}^{b}\left(\tau_{b} ; t\right)$ of size $N \times L$ represent the impulse responses of the forward and backscattering links, respectively. The signaling matrix of the tag, $\mathbf{S}(t)$ with size $L \times L$, describes the time-varying modulation and coding of the carrier signals by the $L$-antenna RF tag. Based on the different tag circuit designs, $\mathbf{S}(t)$ can have several forms: One form is the identical signaling matrix [9]

$$
\mathbf{S}(t)=\Delta(t) \mathbf{I},
$$

where $\Delta(t)$ means the reflection coefficient of the RF tag load, and I means the $L \times L$ identity matrix. In this identical case, all antennas of the RF tag have the same reflection coefficient $\Delta(t)$. Another form is the diagonal signaling matrix [9]

$$
\mathbf{S}(t)=\left(\begin{array}{ccc}
\Delta_{1}(t) & & \\
& \ddots & \\
& & \Delta_{L}(t)
\end{array}\right),
$$

where $\Delta_{l}(t)$ means the load reflection coefficient of the $l$ th tag antenna at time $t$. In this case, the RF tag antennas have different load reflection coefficients. A diagonal signaling matrix with unequal load reflection coefficients may result from space-time-coded tag circuit designs, where the reflection coefficients of different tag antennas are pre-designed according to a certain space-time code.

We consider a general MIMO RFID channel [9] in which the tag is equipped with $L$ antennas, and the reader is equipped with $M$ transmitting antennas that send query signal to the tag and $N$ receiving antennas that receive the signals from the tag, as shown in Figure 2. Since in a passive RFID system, the coding and modulation are done by the tag circuits and the reader transmitting antennas actually act as charging devices by sending (identical) unmodulated waves to the tag, also since the channel gains from reader transmitting antennas to a tag antenna are complex Gaussian distributed, by normalizing the transmitting energy to unity, the statistical property of using a single and multiple reader transmitting antennas are exactly the same. Therefore, we focus on the $1 \times N \times L$ channel in the rest part of the article and we call it the $N \times L$ channel for simplicity. For simplicity of presentation, we also ignore the time index $t$ in the model from now on. Now, considering that $\mathbf{x}$ are unmodulated waves, 


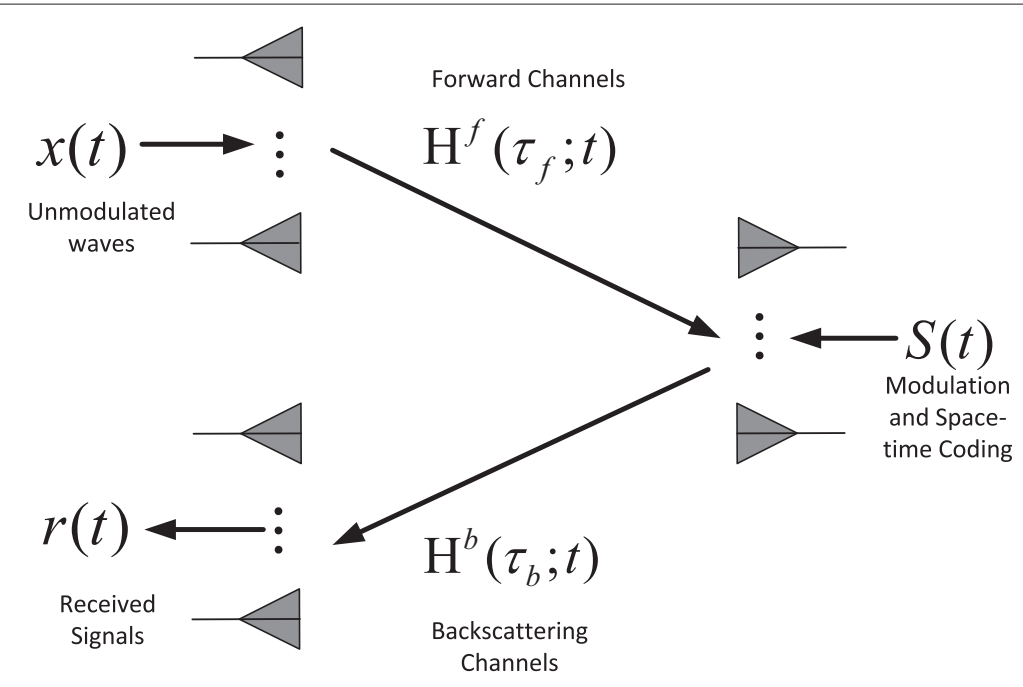

Figure 2 An illustration of the MIMO RFID channel signaling scheme. In a passive RFID system, the coding and modulation are done by the tag circuits at the tag side and the reader transmitting antennas actually act as charging devices. The modulation and coding in the middle way of a cascaded channels make the RFID channel different from other forms of cascaded channels.

let $\mathbf{s}=\left(s_{1}, \ldots, s_{L}\right)^{T}=\left(\Delta_{1}, \ldots, \Delta_{L}\right)^{T}$ denote the $L$ transmission symbols simultaneously transmitted from $L$ tag antennas, we can simply express the received signal vector at a particular time point as

$$
\mathbf{r}=\mathbf{H s}+\mathbf{w}
$$

where the vector $\mathbf{r}=\left(r_{1}, \ldots, r_{N}\right)^{T}$ are the received signals, and each entry of the noise vector $\mathbf{w}=$ $\left(w_{1}, \ldots, w_{N}\right)^{T}$ is assumed to be independent and identical distributed (i.i.d.) Gaussian with zero mean and unit variance. The channel matrix $\mathbf{H}$ of the $N \times L$ channel, which is with size $N \times L$, can be expressed as

$$
\mathbf{H}=\left(\begin{array}{ccc}
h_{1}^{f} h_{1,1}^{b}, h_{2}^{f} h_{2,1}^{b}, & \cdots, & h_{L}^{f} h_{L, 1}^{b} \\
\vdots & \ddots & \vdots \\
h_{1}^{f} h_{1, N}^{b}, h_{2}^{f} h_{2, N}^{b}, & \cdots, & h_{L}^{f} h_{L, N}^{b}
\end{array}\right)
$$

where $h_{l}^{f}$, s $(l=1, \ldots, L)$ represent forward channels of the $N \times L$ channel, $h_{l, n}^{b}$ 's $(l=1, \ldots, L, n=1, \ldots, N)$ represent backscattering channels, and $h_{l}^{f}$, s and $h_{l, n}^{b}$ 's are independent complex Gaussian random variables. The total channel gain at the $n$th receiving antenna is $h_{n}=\sum_{l=1}^{L} h_{l}^{f} h_{l, n}^{b}$. Note that the channel gains $h_{n}$ 's, for $n=1,2, \ldots, N$, contain the common terms $h_{1}^{f}, \ldots, h_{L}^{f}$. It implies that the channel gains at different receiving branches at the reader are not statistically independent with each other.

\section{Difference between the RFID channel and Other types of cascaded channels}

The cascaded Rayleigh channel is also found in the propagation scenarios such as the keyhole propagation [15-17], the double scattering propagation [18] and the amplifyand-forward (AF) relay cooperative communication systems. As shown in Figure 2 we would like to emphasize that the MIMO RFID channel exhibits a different type of cascaded structure rather than that of the keyhole, double scattering, and AF channels due to the modulation and coding that are done at the tag side (middle way of a cascaded channel) rather than the reader transmission side.

For the keyhole channel, the channel matrix is given by $[15,16]$ as

$$
\mathbf{H}_{\text {keyhole }}=\left(\begin{array}{lll}
y_{1} z_{1}, y_{2} z_{1}, & \cdots, & y_{L} z_{1} \\
\vdots & \ddots & \vdots \\
y_{1} z_{N}, y_{2} z_{N}, & \cdots, & y_{L} z_{N}
\end{array}\right)
$$

It is clear that here the rank of the above matrix $\mathbf{H}_{\text {keyhole }}$ is 1 . Comparing with the RFID channel matrix in Equation (5) which is generally full rank, we clearly note that the two channels are different in structure, although for both channels each entry of the matrices is double Rayleigh distributed. Their different channel statistics lead to different performances and diversity gains. The structure of the MIMO RFID channel is also different from that of the double scattering channel in [18], and we will see later in the result part that their performances and diversity gains are different.

Another type of cascaded channel is the AF cooperative channel. For only identical signaling scheme, theoretically it may be possible for an AF model to be reduced to the MIMO RFID model if the AF channel contains $L$ relays 
and has $N$ receiving antennas. A recently published work in [17] discusses the performance of an AF channel with such antenna setting, which is the most similar model to the MIMO RFID channel by far. However, we cannot generalize their results to infer the performance of the MIMO RFID channel. Because in [17], the model is based on a specific transmission scheme in which the relays intentionally forward messages to the destination at different time slots, and the forwarded messages are buffered at the destination, matched filtered, and then combined using maximal ratio combining (MRC). In the MIMO RFID channel, whatever the signaling schemes are used, the signals from the tag antennas (analogy to the relays) arrive at the reader (analogy to the destination) at the same time slot, and this makes the two models significantly different. We will also see differences of these two models from their performances and diversity orders. Other existing references about AF channels either do not consider multiple relays or do not consider multiple receiving antennas and thus cannot be a generalized version of the MIMO RFID channel. Some previous articles consider a single relay with multiple antennas and the model looks similar. However, the model is indeed different (e.g., the relay uses different antennas for transmitting and receiving).

The intention of this study is to analytically derive the performances of the MIMO RFID channel, a specific type of cascaded channel which exists in passive MIMO RFID systems.

\section{Performance analysis of MIMO RFID channel under identical signaling scheme}

In this section, we analytically study BER performances and diversity gains of the $N \times L$ RFID channel when the identical signaling scheme is employed.

Under the identical signaling scheme, the system employs the identical signaling matrix in Equation (2) in which each tag antenna transmits the same symbol at time $t$. From the channel matrix in Equation (5), the channel power at the $n$th receiving branch, also referred as the instantaneous signal-to-noise ratio (SNR) at the $n$th receiving antenna, can be given by

$$
\gamma_{n}=\bar{\gamma}\left|\sum_{l=1}^{L} h_{l}^{f} h_{l, n}^{b}\right|^{2}
$$

where $\bar{\gamma}$ means the average SNR. Each of these instantaneous SNRs follows the following distribution [9]

$$
f_{\gamma_{n}}\left(\gamma_{n}\right)=\frac{2 \gamma_{n}^{(L-1) / 2}}{(L-1) ! \bar{\gamma}^{(L+1) / 2}} K_{L-1}\left(2 \sqrt{\frac{\gamma_{n}}{\bar{\gamma}}}\right)
$$

where $K_{L-1}(\cdot)$ denotes the modified Bessel function of the second kind. Using the asymptotic approximations of the Bessel function [19], one can obtain the approximation of the PDF for high SNR (e.g., as $\bar{\gamma} \rightarrow \infty$ ) as,

$$
f_{\gamma_{n}}\left(\gamma_{n}\right) \doteq \begin{cases}-\frac{1}{\bar{\gamma}} \ln \left(\frac{\gamma_{n}}{\bar{\gamma}}\right), & \text { if } L=1 ; \\ \frac{1}{(L-1) \bar{\gamma}}, & \text { if } L>1 .\end{cases}
$$

To derive the BER performance of the $N \times L$ MIMO RFID channel where $N>1$, since the $N$ receiving branches at the reader are not statistically independent as we mentioned earlier, we cannot use the above distribution and its approximation to directly evaluate the performance of the MIMO channel by applying a widely used method as in $[20,21]$ which assumes independency of receiving branches. Alternatively, we consider evaluating the BER using the conditional probability approach. We will see later, to analytically study the BER performance, we first need to investigate the properties of $G_{N, L}(\cdot)$, a function defined by a multi-variate integration. The function $G_{N, L}(\cdot)$ is defined as

$$
\begin{aligned}
G_{N, L}(\overline{\bar{\gamma}})= & \int_{\alpha_{L}=0}^{\infty} \cdots \int_{\alpha_{1}=0}^{\infty} \frac{1}{\left(1+\overline{\bar{\gamma}} \sum_{l=1}^{L} \alpha_{l}\right)^{N}} \\
& \times \exp \left(-\sum_{l=1}^{L} \alpha_{l}\right) d \alpha_{1} \cdots d \alpha_{L} .
\end{aligned}
$$

Here $\alpha_{l}$ is the squared magnitude of the channel gain of the $l$ th receiving branch, $N$ and $L$ are the index of the function $G_{N, L}(\overline{\bar{\gamma}})$, and we define $\overline{\bar{\gamma}}=\frac{g \bar{\gamma}}{\sin ^{2} \theta}$, where $\bar{\gamma}$ is the average SNR and $g$ is a constant which is modulation dependent. For the coherent transmission case, the function $G_{N, L}(\overline{\bar{\gamma}})$ is the moment generating function (MGF) of the MIMO RFID channel with $L$ tag antennas and $N$ receiving antennas. For the non-coherent transmission case, the form of $G_{N, L}(\cdot)$ is required in deriving the BER performance. The function $G_{N, L}(\cdot)$ defined in Equation (10) has the following recursive and asymptotic properties

\section{Proposition 1.}

$$
G_{1, L}(\overline{\bar{\gamma}})=\frac{e^{\frac{1}{\bar{\gamma}}}}{\overline{\bar{\gamma}}} E_{L}\left(\frac{1}{\overline{\bar{\gamma}}}\right) \doteq \begin{cases}\frac{\ln (\overline{\bar{\gamma}})}{\overline{\bar{\gamma}}}, & \text { if } L=1 \\ \frac{1}{(L-1) \overline{\bar{\gamma}}}, & \text { if } L>1\end{cases}
$$




\section{Proposition 2.}

$$
G_{N, 1}(\overline{\bar{\gamma}})=\frac{e^{\frac{1}{\bar{\gamma}}}}{\overline{\bar{\gamma}}} E_{N}\left(\frac{1}{\overline{\bar{\gamma}}}\right) \doteq \begin{cases}\frac{\ln (\overline{\bar{\gamma}})}{\overline{\bar{\gamma}}}, & \text { if } N=1 \\ \frac{1}{(L-1) \overline{\bar{\gamma}}}, & \text { if } N>1\end{cases}
$$

\section{Proposition 3.}

$$
\begin{aligned}
G_{N, L}(\overline{\bar{\gamma}})= & \frac{1}{(-\overline{\bar{\gamma}})^{N-1}(N-1) !} G_{1, L}(\overline{\bar{\gamma}})-\sum_{k=1}^{N-1} \frac{(k-1) !}{(-\overline{\bar{\gamma}})^{N-k}(N-1) !} \\
& \times G_{k,(L-1)}(\overline{\bar{\gamma}}) .
\end{aligned}
$$

\section{Proposition 4.}

$$
G_{N, L}(\overline{\bar{\gamma}}) \doteq \begin{cases}\frac{1}{(L-1) \cdots(L-N) \overline{\bar{\gamma}}^{N}}, & \text { if } N<L ; \\ \frac{\ln (\overline{\bar{\gamma}})}{(N-1) ! \overline{\bar{\gamma}}^{N}}, & \text { if } N=L ; \\ \frac{1}{(N-1) \cdots(N-L) \overline{\bar{\gamma}}^{L}}, & \text { if } N>L\end{cases}
$$

In the above propositions, $E_{N}(\cdot)$ and $E_{L}(\cdot)$ are the exponential integrals defined as $E_{N}(x)=\int_{t=1}^{\infty} \frac{\exp (-t x)}{t^{N}} d x$ and $E_{L}(x)=\int_{t=1}^{\infty} \frac{\exp (-t x)}{t^{L}} d x$ [22], where $N$ and $L$ are positive integers. The proofs of these propositions can be found in Appendix. With the above properties, we are now ready to derive the exact and asymptotic BER performances and study how the MIMO RFID backscattering channel behaves.

\section{Non-coherent Case}

We first investigate the non-coherent transmission case, which applies non-coherent equal gain combining (EGC) at the reader receiver side.

The channel gain at the $n$th receiving branch of the reader is given by $h_{n}=\sum_{l=1}^{L} h_{l}^{f} h_{l, n}^{b}$. When fixing the forward channel gains $h_{l}^{f}$ s, the channel gain $h_{n}$ is a linear combination of i.i.d. complex Gaussian random variables, hence the conditional distribution of $h_{n}$ on $h_{l}^{f}$, s is a complex Gaussian distribution with variance $\sum_{l=1}^{L}\left|h_{l}^{f}\right|^{2}$. Therefore, by fixing $h_{l}^{f}$, s, the $N \times L$ channel can be viewed as a single-input multiple-output (SIMO) channel in which each receiving branch is Rayleigh distributed and has power (or variance) $\sum_{l=1}^{L}\left|h_{l}^{f}\right|^{2}$. Consequently using the result of the SIMO Rayleigh channel [23], we can have the conditional (on $h_{l}^{f}$, s) BER for the $N \times L$ RFID channel using non-coherent EGC as

$$
\begin{aligned}
P_{N, L}\left(\bar{\gamma} \mid h_{l}^{f}\right)= & \frac{1}{2^{2 N-1}(N-1) !\left(1+g \bar{\gamma} \sum_{l=1}^{L}\left|h_{l}^{f}\right|^{2}\right)^{N}} \\
& \sum_{k=0}^{N-1} b_{k}(N-1+k) !\left(\frac{g \bar{\gamma} \sum_{l=1}^{L}\left|h_{l}^{f}\right|^{2}}{1+g \bar{\gamma}\left(\sum_{l=1}^{L}\left|h_{l}^{f}\right|^{2}\right)}\right)^{k},
\end{aligned}
$$

where $b_{k}=\frac{1}{k !} \sum_{n=0}^{N-1-k}\left(\begin{array}{l}2 N-1) \\ n\end{array}\right.$ and $g$ is a constant which is modulation dependent [24]. Note that $\left(\frac{g \bar{\gamma} \sum_{l=1}^{L}\left|h_{l}^{f}\right|^{2}}{1+g \bar{\gamma} \sum_{l=1}^{L}\left|h_{l}^{f}\right|^{2}}\right)^{k}=\left(1-\frac{1}{1+g \bar{\gamma} \sum_{l=1}^{L}\left|h_{l}^{f}\right|^{2}}\right)^{k}=\sum_{i=0}^{k}(-1)^{i}$ $\left(\begin{array}{c}k \\ i\end{array}\right) \frac{1}{\left(1+g \bar{\gamma} \sum_{l=1}^{L}\left|h_{l}^{f}\right|^{2}\right)^{i}}$, then we have

$$
\begin{aligned}
P_{N, L}\left(\bar{\gamma} \mid h_{l}^{f}\right)= & \frac{1}{2^{2 N-1}(N-1) !} \sum_{k=0}^{N-1} b_{k}(N-1+k) ! \sum_{i=0}^{k}(-1)^{i} \\
& \times\left(\begin{array}{l}
k \\
i
\end{array}\right) \frac{1}{\left(1+g \bar{\gamma} \sum_{l=1}^{L}\left|h_{l}^{f}\right|^{2}\right)^{N+i}}
\end{aligned}
$$

Averaging the conditional BER over $\alpha_{l}$ 's (where $\left|h_{l}^{f}\right|^{2}=$ $\alpha_{l}$ ) yields the BER for the $N \times L$ RFID channel as

$$
\begin{aligned}
P_{N, L}(\bar{\gamma})= & \int_{\alpha_{L}=0}^{\infty} \cdots \int_{\alpha_{1}=0}^{\infty} P_{N, L}\left(\bar{\gamma} \mid \alpha_{l}\right) \\
& \times \exp \left(-\sum_{l=1}^{L} \alpha_{l}\right) d \alpha_{1} \cdots \alpha_{L} \\
= & \frac{1}{2^{2 N-1}(N-1) !} \sum_{k=0}^{N-1} b_{k}(N-1+k) ! \sum_{i=0}^{k}(-1)^{i} \\
& \times\left(\begin{array}{c}
k \\
i
\end{array}\right) G_{(N+i), L}(\bar{\gamma}) .
\end{aligned}
$$

The closed-form of the above exact BER can be computed recursively using Proposition 3 with the initial knowledge $G_{1, L}(\bar{\gamma})=\frac{e^{\frac{1}{\gamma}}}{\bar{\gamma}} E_{L}(\bar{\gamma})$ and $G_{N, 1}(\bar{\gamma})=\frac{e^{\frac{1}{\bar{\gamma}}}}{\bar{\gamma}} E_{N}(\bar{\gamma})$. Table 1 shows a few examples under some antenna settings.

While the closed-form BER can be obtained, it involves complicated recursive forms and the behavior of the stud$\operatorname{ied} N \times L$ RFID channel is not easy to analyze, and we need to investigate an asymptotic form. Using Proposition 4, we can obtain an asymptotic BER of Equation (17) as 


$$
P_{N, L}(\bar{\gamma}) \doteq \begin{cases}\frac{\sum_{k=0}^{N-1} b_{k}(N-1+k) !}{2^{2 N-1}(N-1) !} G_{N, L}(g \bar{\gamma}) \doteq \frac{\sum_{k=0}^{N-1} b_{k}(N-1+k) !}{2^{2 N-1}(N-1) !(L-1) \cdots(L-N)(g \bar{\gamma})^{N}}, & \text { if } N<L ; \\
\frac{\sum_{k=0}^{N-1} b_{k}(N-1+k) !}{2^{2 N-1}(N-1) !} G_{N, L}(\bar{\gamma}) \doteq \frac{\sum_{k=0}^{N-1} b_{k}(N-1+k) ! \ln (g \bar{\gamma})}{2^{2 N-1}(N-1) !(N-1) !(g \bar{\gamma})^{N}}, & \text { if } N=L ; \\
\frac{1}{2^{2 N-1}(N-1) !} \sum_{k=0}^{N-1} b_{k}(N-1+k) ! \sum_{i=0}^{k}(-1)^{i}\left(\begin{array}{l}
k \\
i
\end{array}\right) \frac{1}{(N+i-1) \cdots(N+i-L)(g \bar{\gamma})^{L}}, & \text { if } N>L .\end{cases}
$$

We can see that the above asymptotic BER form depends on the relation of the values of $L$ and $N$. Figure 3 shows the BER performances of the $N \times L$ RFID channels when employing the binary frequency-shift keying (FSK) with EGC.

The asymptotic diversity order $d_{a}$ can be obtained as

$$
d_{a}=\lim _{\bar{\gamma} \rightarrow \infty}\left(-\frac{\log P_{N, L}(\bar{\gamma})}{\log (\bar{\gamma})}\right)=\min (N, L)
$$

It means that the asymptotic diversity order of the $N \times L$ RFID channel under non-coherent transmission schemes is determined by the smaller value of $N$ and $L$. For the case of $L=N$, compared with the case of $L \neq N$, it requires a higher SNR to achieve the diversity order $N$, because of the logarithm function in the numerator in Equation (18) when $N=L$. This property means that even the diversity orders are the same the BER performances of the settings with $N=L+1$ or $L=N+1$ are remarkably better than the performance of the setting with $N=L$. The BER performance improvements from $N=L+1$ to $N=L+2$, or from $L=N+1$ to $L=N+2$, is not significant. These observations generalize the findings about the MISO case in [25].

\section{Coherent case}

We now look at the coherent detection case. We assume that the reader knows the channel state information (CSI) and MRC is applied at the reader receiver side.

Similar to our previous derivation, if we fix the forward gains $h_{l}^{f}$,s, the MIMO RFID channel can be viewed as a SIMO Rayleigh channel in which the receiving branches are independent and have power (or variance) $\sum_{l=1}^{L}\left|h_{l}^{f}\right|^{2}$. Recall that the MGF for a Rayleigh fading channel is given by $\left(1+\frac{g \bar{\gamma}}{\sin ^{2} \theta}\right)^{-1}$ [20]; therefore, the conditional MGF of the $N \times L$ RFID backscattering channel is

$$
M_{N, L}\left(g, \bar{\gamma}, \theta \mid h_{l}^{f}\right)=\left(1+\frac{g \bar{\gamma} \sum_{l=1}^{L}\left|h_{l}^{f}\right|^{2}}{\sin ^{2} \theta}\right)^{-N} .
$$

Integrating $M_{N, L}\left(g, \bar{\gamma}, \theta \mid h_{l}^{f}\right)$ over $\alpha_{l}$ 's (where $\left.\alpha_{l}=\left|h_{l}^{f}\right|^{2}\right)$ leads to the MGF for non-independent $N$ receiving branches as

$$
\begin{aligned}
M_{N, L}(g, \theta, \bar{\gamma})= & \int_{\alpha_{L}=0}^{\infty} \cdots \int_{\alpha_{1}=0}^{\infty} M_{N, L}\left(g, \theta, \bar{\gamma} \mid \alpha_{l}\right) \\
& \times \exp \left(-\sum_{l=1}^{L} \alpha_{l}\right) d \alpha_{1} \cdots d \alpha_{L} \\
= & \int_{\alpha_{L}=0}^{\infty} \cdots \int_{\alpha_{1}=0}^{\infty}\left(1+\frac{g \bar{\gamma} \sum_{l=1}^{L} \alpha_{l}}{\sin ^{2} \theta}\right)^{-N} \\
& \times \exp \left(-\sum_{l=1}^{L} \alpha_{i}\right) d \alpha_{1} \cdots d \alpha_{L}=G_{N, L}(\overline{\bar{\gamma}}),
\end{aligned}
$$

where $\overline{\bar{\gamma}}=\frac{g \bar{\gamma}}{\sin ^{2} \theta}$ and $G_{N, L}(\cdot)$ is defined as in Equation (10). Using the moment generating approach in [20], the BER of the $N \times L$ RFID channel for the coherent case can be expressed as

$$
P_{N, L}(\bar{\gamma})=\frac{1}{\pi} \int_{\theta=0}^{\pi / 2} G_{N, L}(\overline{\bar{\gamma}}) d \theta .
$$

Table 2 shows a few examples of the closed form of the MGF of the channel with coherent transmission scheme. Since the closed form of $G_{N, L}(\overline{\bar{\gamma}})$ can be obtained recursively using Propositions 1 to 4 , the BER $P_{N, L}(\bar{\gamma})$ can be computed through the single integration in Equation (22) respective to $\theta$. To have more insights on how the BER of the $N \times L$ RFID channel behaves, we also derive an asymptotic form of this BER expression. Using Proposition 4, the asymptotic BER for Equation (22) can be expressed as

$P_{N, L}(\bar{\gamma}) \doteq \begin{cases}\frac{1}{\pi} \int_{\theta=0}^{\pi / 2} \frac{1}{(L-1) \cdots(L-N)(g \overline{\bar{\gamma}})^{N}} d \theta=\frac{C_{N}}{(L-1) \cdots(L-N)(g \bar{\gamma})^{N}}, & \text { if } N<L ; \\ \frac{1}{\pi} \int_{\theta=0}^{\pi / 2} \frac{\ln (g \bar{\gamma})}{(N-1) !(g \bar{\gamma})^{N}} d \theta \doteq \frac{C_{N} \ln (g \bar{\gamma})}{(N-1) !(g \bar{\gamma})^{N}}, & \text { if } N=L ; \\ \frac{1}{\pi} \int_{\theta=0}^{\pi / 2} \frac{1}{(N-1) \cdots(N-L)(g \bar{\gamma})^{L}} d \theta=\frac{C_{L}}{(N-1) \cdots(N-L)(g \bar{\gamma})^{L}}, & \text { if } N \geq L,\end{cases}$

where $C_{N}=\int_{\theta=0}^{\pi / 2} \sin ^{2 N} \theta d \theta=\frac{\Gamma(1 / 2+N)}{2 \sqrt{\pi} \Gamma(1+N)}$ and $C_{L}=\frac{\Gamma(1 / 2+L)}{2 \sqrt{\pi} \Gamma(1+L)}$. Here $\Gamma(\cdot)$ means the Gamma function. Figure 4 plots the BER curves of the $N \times L$ RFID channels when employing BPSK with MRC at the reader receiver antennas.

For the $N \times L$ RFID channel under the coherent case, the asymptotic diversity order can be given by

$$
d_{a}=\lim _{\bar{\gamma} \rightarrow \infty}\left(-\frac{\log P_{N, L}(\bar{\gamma})}{\log (\bar{\gamma})}\right)=\min (N, L) .
$$


Table 1 Non-coherent case of the identical signaling scheme: closed-form BER expressions for the $N \times L R F I D$ channel (Equation 17)

\begin{tabular}{|c|c|c|}
\hline & $L=1$ & $L=2$ \\
\hline$N=1$ & $e^{\frac{1}{g \hat{\gamma}}} \frac{E_{1}\left(\frac{1}{g \bar{\gamma}}\right)}{2 g \bar{\gamma}}$ & $e^{\frac{1}{g \hat{\gamma}}} \frac{E_{2}\left(\frac{1}{g \bar{\gamma}}\right)}{2 g \bar{\gamma}}$ \\
\hline \multirow{3}{*}{$N=2$} & 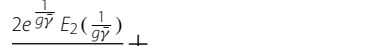 & $2\left(-g \bar{\gamma}+e^{\frac{1}{g \gamma}} E_{1}\left(\frac{1}{g \bar{\gamma}}\right)+g \bar{\gamma}\right)$ \\
\hline & $\begin{array}{l}g \bar{\gamma} \\
(g \bar{\gamma})^{2}-2 g \bar{\gamma} e^{\frac{1}{g \gamma}} E_{1}\left(\frac{1}{g \bar{\gamma}}\right)+g \bar{\gamma}-e^{\frac{1}{g \gamma}} E_{1}\left(\frac{1}{g \bar{\gamma}}\right)\end{array}$ & $\begin{array}{c}g \bar{\gamma} \\
e^{\frac{1}{g \gamma}} E_{1}\left(\frac{1}{g \bar{\gamma}}\right)-3(g \bar{\gamma})^{2}+2(g \bar{\gamma})^{2} e^{\frac{1}{g \bar{\gamma}}} E_{1}\left(\frac{1}{g \bar{\gamma}}\right)-g \bar{\gamma}+4 g \bar{\gamma} e^{\frac{1}{g \gamma}} E_{1}\left(\frac{1}{g \bar{\gamma}}\right)\end{array}$ \\
\hline & $4(g \bar{\gamma})^{3}$ & $4(g \bar{\gamma})^{3}$ \\
\hline
\end{tabular}

As we can see that the asymptotic diversity order is still $\min (N, L)$ in the coherent transmission case, and the BER behavior is similar to that of the non-coherent case.

\section{Performance analysis of MIMO RFID channel under OSTBC scheme}

In this section, we analytically study BER performances and diversity gains of the $N \times L$ RFID channel when the orthogonal space-time coding scheme (OSTBC) is employed. OSTBC is one of the most attractive MIMO schemes with a very simple decoding process based on linear combining at the receiver. Because of its orthogonality, OSTBC achieves full diversity $L N$ for $L$ transmission antennas and $N$ receiving antennas in i.i.d. MIMO Rayleigh fading channels [26,27]. In this section, we investigate the error rate performance of the MIMO RFID channel using the OSTBC scheme. For MIMO RFID systems, OSTBC can be implemented by applying the signaling matrix in Equation(3) for the tag antennas. We assume that CSI is known by the reader and the channel is quasi-static.
Because of its orthogonality property, OSTBC can transform from the MIMO fading channel

$$
\mathbf{r}=\mathbf{H s}+\mathbf{w},
$$

to the following parallel SISO channels [28]

$$
\mathbf{r}^{\prime}=\sqrt{\frac{\|\mathbf{H}\|^{2}}{L}} \mathbf{s}^{\prime}+\mathbf{w}^{\prime},
$$

where

$$
\|\mathbf{H}\|=\sqrt{\sum_{n=1}^{N} \sum_{l=1}^{L}\left|h_{l}^{f} h_{l, n}^{b}\right|^{2}}
$$

is the Frobenius norm of $\mathbf{H}, \mathbf{s}=\left(s_{1}^{\prime}, \ldots, s_{Q}^{\prime}\right)^{T}$ is the $Q$ incoming symbols and the entries of $\mathbf{w}^{\prime}=$ $\left(w_{1}^{\prime}, \ldots, w_{Q}^{\prime}\right)^{T}$ are i.i.d. Complex Gaussian random variables with zero mean and unit variance. Similarly, the entries of $\mathbf{r}^{\prime}=\left(r_{1}^{\prime}, \ldots, r_{Q}^{\prime}\right)^{T}$ are the receiving symbols and can be detected based on a simple maximum likelihood method. The channel gain is divided by $L$ because the transmission power should be normalized to unity. Since

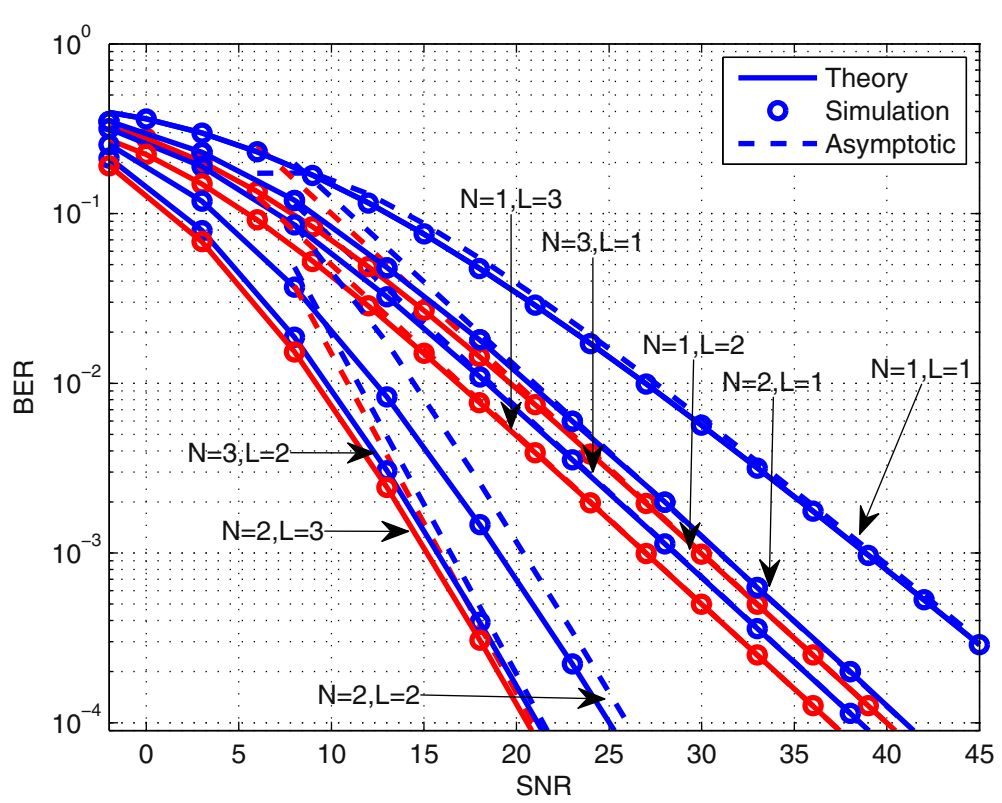

Figure 3 BER performances (in Equations 17 and 18) of the MIMO RFID channel using non-coherent identical signaling (BPSK with EGC). 
Table 2 Coherent case of the identical signaling scheme: MGFs $G_{N, L}(\theta)$ for the $N \times L$ RFID channel (Equation 21)

$\begin{array}{ll}\boldsymbol{L}=\mathbf{1} & \boldsymbol{L}=\mathbf{2} \\ N=1 e^{\frac{\sin ^{2} \theta}{g \bar{\gamma}}} \frac{E_{1}\left(\frac{\sin ^{2} \theta}{g \bar{\gamma}}\right)}{g \bar{\gamma}} & \frac{e^{\frac{\sin ^{2} \theta}{g \bar{\gamma}}} E_{2}\left(\frac{\sin ^{2} \theta}{g \bar{\gamma}}\right) \sin ^{2} \theta}{g \bar{\gamma}} \\ N=2 \frac{e^{\frac{\sin ^{2} \theta}{g \bar{\gamma}}} E_{2}\left(\frac{\sin ^{2} \theta}{g \bar{\gamma}}\right) \sin ^{2} \theta}{g \bar{\gamma}} & \frac{-\bar{\gamma} \sin ^{4} \theta+e^{\frac{\sin ^{2} \theta}{g \bar{\gamma}}} E_{1}\left(\frac{\sin ^{2} \theta}{g \bar{\gamma}}\right) \sin ^{6} \theta+\bar{\gamma} e^{\frac{\sin ^{2} \theta}{g \bar{\gamma}}} E_{1}\left(\frac{\sin ^{2} \theta}{g \bar{\gamma}}\right) \sin ^{4} \theta}{(g \bar{\gamma})^{3}}\end{array}$

in real passive RFID signal transmissions, the transmission energy is from the reader and is proportional to the number of tag antennas when the reader querying energy is fixed, we instead use the following model

$$
\mathbf{r}^{\prime}=\sqrt{\|\mathbf{H}\|^{2}} \mathbf{s}^{\prime}+\mathbf{w}^{\prime}
$$

in which the transmission power is not normalized to unity. Let $E_{b}$ denote the average energy per bit and $E_{s}$ denote the average energy per symbol then $E_{s}=E_{b} \log _{2} K$ where $K$ is the size of the signal constellation. The instantaneous SNR per symbol is therefore given by

$$
\gamma=\frac{\|\mathbf{H}\|^{2} \log _{2} K}{R} \frac{E_{b}}{N_{0}}=\frac{\|\mathbf{H}\|^{2} \log _{2} K}{R} \bar{\gamma}=\|\mathbf{H}\|^{2} g \bar{\gamma}
$$

where $R$ means the symbol rate and we define $g=\frac{\log _{2} K}{R}$.

Since evaluating the density of $\|\mathbf{H}\|^{2}$ directly is difficult, we consider the conditional probability approach as in previous derivations. Define $\left|\mathbf{H}_{l}\right|^{2}=\sum_{n=1}^{N} \alpha_{l} \beta_{l, n}$, where $\alpha_{l}=\left|h_{l}^{f}\right|^{2}$ and $\beta_{l, n}=\left|h_{l, n}^{b}\right|^{2}$. It is easy to check that $\left|\mathbf{H}_{l}\right|^{\prime}$ are i.i.d. random variables. Because $\|\mathbf{H}\|^{2}=$
$\sum_{l=1}^{L}\left|\mathbf{H}_{l}\right|^{2}$, for the SER analysis we can view the $N \times L$ RFID channel which uses OSTBC as a virtual SIMO system with $L$ receiving branches in which MRC is applied at the receiver side. For the $l$ th receiving branch of this SIMO system, the squared magnitude of the channel gain is $\left|\mathbf{H}_{l}\right|^{2}=\sum_{n=1}^{N} \alpha_{l} \beta_{l, n}$, where $\alpha_{l}$ is the channel power at the $l$ th receiving branch. We define the MGF of $\left|\mathbf{H}_{l}\right|^{2}$ as $G_{l}(\overline{\bar{\gamma}})$, where $\overline{\bar{\gamma}}=\frac{g \bar{\gamma}}{\sin ^{2} \theta}$, then the BER can be evaluated via

$$
P(\bar{\gamma})_{N, L}=\frac{1}{\pi} \int_{\theta=0}^{\pi / 2} \prod_{l=1}^{L} G_{l}(\overline{\bar{\gamma}}) d \theta .
$$

Now we need to derive $G_{l}(\overline{\bar{\gamma}})$. Since the conditional channel gain $\left|\mathbf{H}_{l}\right|^{2}=\sum_{n=1}^{N} \alpha_{l} \beta_{l, n}$ given $\alpha_{l}$ can be viewed as the channel gain of another virtual SIMO system (i.e., $N$ Rayleigh distributed receiving branches with gains $h_{l, n}^{b}$ 's, $n=1, \ldots, N)$ in which MRC is applied at the receiver side, consequently the MGF of $\left|\mathbf{H}_{l}\right|$ can be derived by averaging the conditional MGF $G_{l}\left(\overline{\bar{\gamma}} \mid \alpha_{l}\right)$ over $\alpha_{l}$, with $G_{l}\left(\overline{\bar{\gamma}} \mid \alpha_{l}\right)=$ $\left(1+\alpha_{l} \overline{\bar{\gamma}}\right)^{-N}$ being the MGF of an $N$-branches Rayleigh channel [20]

$$
\begin{aligned}
G_{l}(\overline{\bar{\gamma}}) & =\int_{\alpha_{l}=0}^{\infty} G_{l}\left(\overline{\bar{\gamma}} \mid \alpha_{l}\right) f_{\alpha_{l}}\left(\alpha_{l}\right) d \alpha_{l} \\
& =\int_{\alpha_{l}=0}^{\infty}\left(1+\alpha_{l} \overline{\bar{\gamma}}\right)^{-N} \exp \left(-\alpha_{l}\right) d \alpha_{l} \\
& =\frac{e^{\frac{1}{\bar{\gamma}}}}{\overline{\bar{\gamma}}} E_{N}\left(\frac{1}{\overline{\bar{\gamma}}}\right) \doteq \begin{cases}\frac{\ln (\overline{\bar{\gamma}})}{\overline{\bar{\gamma}}}, & \text { if } N=1 ; \\
\frac{1}{(N-1) \overline{\bar{\gamma}}}, & \text { if } N>1 .\end{cases}
\end{aligned}
$$

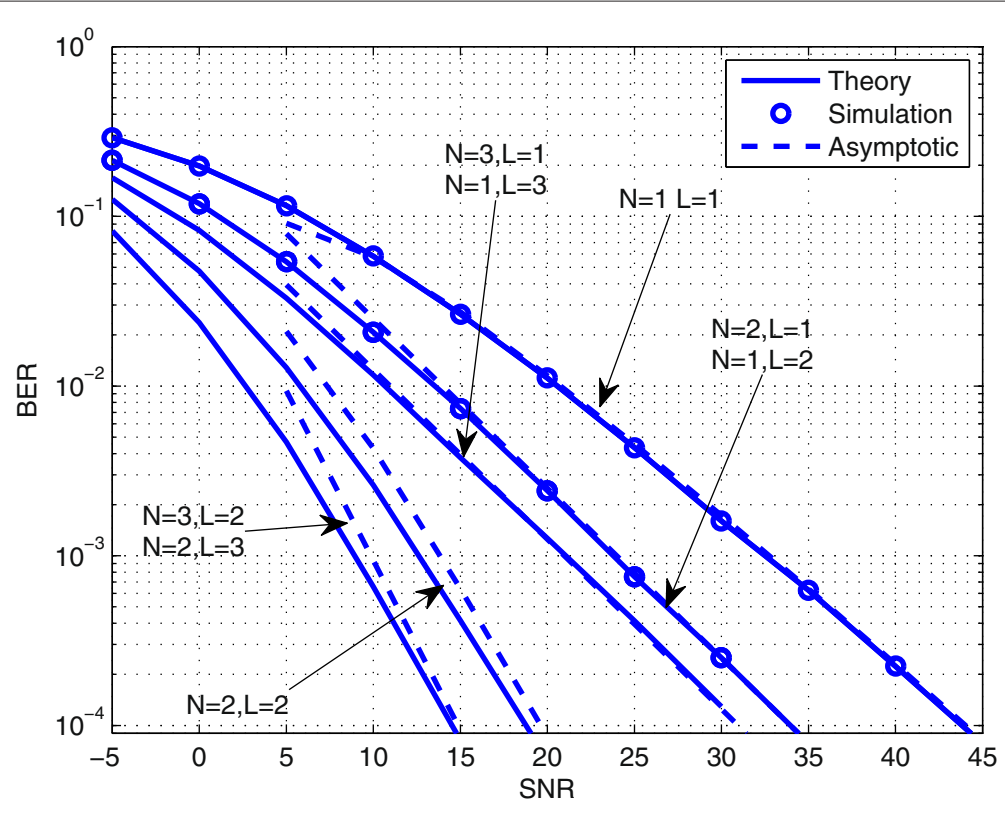

Figure 4 BER performances (in Equations 22 and 23) of the MIMO RFID channel using coherent identical signaling (BPSK with MRC) under perfect channel estimation. 
Substituting $G_{l}(\overline{\bar{\gamma}})$ into Equation (30) yields the BER expression of the $N \times L$ RFID channel for the OSTBC scheme

$$
\begin{aligned}
P_{N, L}(\bar{\gamma}) & =\frac{1}{\pi} \int_{\theta=0}^{\frac{\pi}{2}}\left(e \frac{g \bar{\gamma}}{\sin ^{2} \theta} \sin ^{2} \theta \frac{E_{N}\left(\frac{\sin ^{2} \theta}{g \bar{\gamma}}\right)}{g \bar{\gamma}}\right)^{L} d \theta \\
& \doteq \begin{cases}C_{L}\left(\frac{\ln (g \bar{\gamma})}{g \bar{\gamma}}\right)^{L} & \text { if } N=1 ; \\
C_{L}\left(\frac{1}{(N-1) g \bar{\gamma}}\right)^{L}, & \text { if } N>1 .\end{cases}
\end{aligned}
$$

Figure 5 shows the SER/BER curves of Alamouti's code with BPSK modulation in the $N \times L$ MIMO RFID channel (the SER and BER are exactly same for Alamouti's code using BPSK).

Accordingly, for the $N \times L$ RFID channel with the OSTBC scheme, the asymptotic diversity order can be derived as

$$
d_{a}=\lim _{\bar{\gamma} \rightarrow \infty}\left(-\frac{\log P_{N, L}(\bar{\gamma})}{\log (\bar{\gamma})}\right)=L .
$$

From Equation (34) we can see that here the achievable diversity order is $L$ (the number of tag antennas), and $N$ (the number of reader receiving antennas) does not affect the diversity gain. However, we note that there is a significant BER improvement from $N=1$ to $N>1$ due to the logarithm function in Equation (33) when $N=1$. This property is very different from that of traditional one-way channels in $[27,29]$ and even very different from independent double Rayleigh channels [30], as we will see in comparisons in Table 3.

\section{Results and discussions}

In this section, we compare the error rate performances of the MIMO RFID backscattering channel with other forms of cascaded channels: the keyhole channel, the double scattering channel, and the independent double Rayleigh channel. We also compare the identical signaling scheme and the OSTBC scheme in the MIMO RFID channel and discuss how much improvement can be achieved by the OSTBC scheme under different antennas settings.

The pair-wise error probability for another cascaded channel which has independent receiving branches and the channel matrix of the following form

$$
\mathbf{H}_{\text {indep }}=\left(\begin{array}{lll}
y_{1,1} z_{1,1}, y_{2,2} z_{2,2}, & \cdots, & y_{L, L} z_{L, L} \\
\vdots & \ddots & \vdots \\
y_{N, 1} z_{N, 1}, y_{N, 2} z_{N, 2}, & \cdots, & y_{N, L} z_{N, L}
\end{array}\right)
$$

was discussed in [30] for space-time trellis code; however, the performance of the identical signal scheme of this channel has not been studied yet. In this section, we give out its BER performance for comparison purposes. For

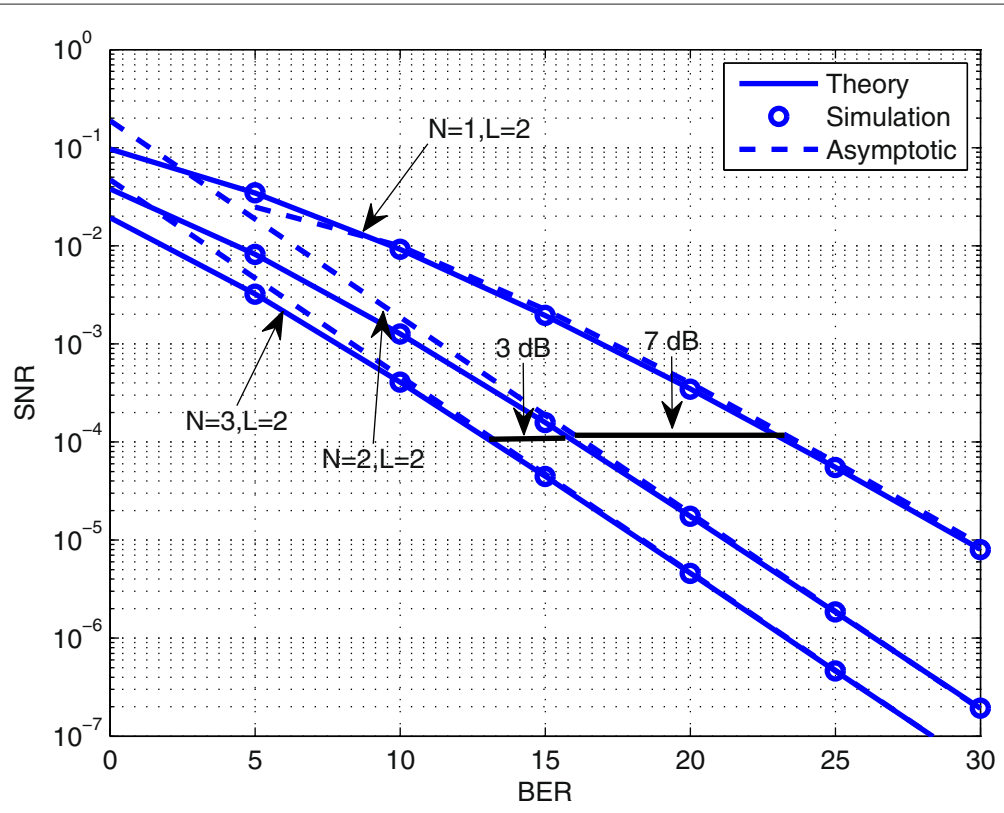

Figure 5 The SER/BER performances (in Equations 32 and 33) of the Alamouti's code with BPSK modulation and MRC in the MIMO RFID channel under perfect channel estimation. 
Table 3 Diversity order comparisons between different cascaded structures

\begin{tabular}{lll}
\hline Channel and coding scheme & Diversity order & Performance bottleneck \\
\hline RFID (identical) & $\min (L, N)$ & $|L-N|>1$ \\
RFID (OSTBC) & $L$ & $N>2$ \\
Keyhole channel (OSTBC) & $\min (L, N)[16]$ & $|L-N|>1$ \\
Double scattering channel (OSTBC) & $N L N / \max (M, L, N)[18]$ & - \\
Independent Double Rayleigh (identical) & $N$ & $L>2$ \\
Independent Double Rayleigh (OSTBC) & $N L[30]$ & No bottleneck
\end{tabular}

non-coherent identical signaling transmission with EGC, the exact BER is given by

$$
\begin{aligned}
P_{N, L}(\bar{\gamma})= & \frac{1}{2^{2 N-1}} \sum_{k=0}^{N-1} b_{k} \sum_{\mathbf{m} \in \mathbb{M}_{k}}\left(\begin{array}{l}
k \\
\mathbf{m}
\end{array}\right) \prod_{n=1}^{N} \frac{\Gamma\left(L+m_{n}\right) \Gamma\left(1+m_{n}\right)}{\Gamma(L) g \bar{\gamma}} \\
& \times U\left(L+m_{n}, L ; \frac{1}{g \bar{\gamma}}\right),
\end{aligned}
$$

where $U(a, b, x)$ is the confluent hypergeometric function, and the corresponding asymptotic BER expression

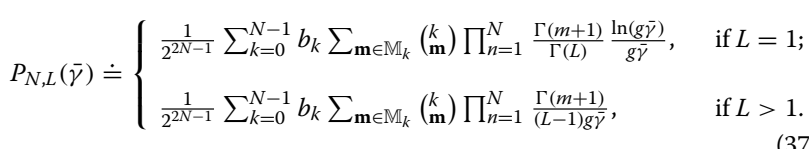

For coherent identical signaling transmission with MRC under perfect channel estimation, the exact and asymptotic BERs are given by

$$
\begin{aligned}
P_{N, L}(\bar{\gamma})= & \frac{1}{\pi} \int_{\theta=0}^{\pi / 2}\left(G_{1, L}(\overline{\bar{\gamma}})\right)^{N} d \theta=\frac{1}{\pi} \int_{\theta=0}^{\pi / 2} \\
& \times\left(\frac{e^{\frac{1}{\bar{\gamma}}}}{\overline{\bar{\gamma}}} E_{L}\left(\frac{1}{\overline{\bar{\gamma}}}\right)\right)^{N} d \theta \\
\doteq & \begin{cases}\frac{1}{\pi} \int_{\theta=0}^{\pi / 2}\left(\frac{\ln (\overline{\bar{\gamma}})}{\overline{\bar{\gamma}}}\right)^{N} d \theta=C_{N}\left(\frac{\ln (\bar{\gamma})}{\bar{\gamma}}\right)^{N}, \quad \text { if } L=1 ; \\
\frac{1}{\pi} \int_{\theta=0}^{\pi / 2}\left(\frac{1}{(L-1) \overline{\bar{\gamma}}}\right)^{N} d \theta=C_{N}\left(\frac{1}{(L-1) \bar{\gamma}}\right)^{N}, \text { if } L>1 .\end{cases}
\end{aligned}
$$

The derivations of the above results are given in Appendix.

Accordingly, we can show that the diversity order under both transmission schemes is

$$
d_{a}=\lim _{\bar{\gamma} \rightarrow \infty}\left(-\frac{\log P_{N, L}(\bar{\gamma})}{\log (\bar{\gamma})}\right)=N .
$$

Figure 6 and Figure 7 show the BER curve for the independent double Rayleigh channel with non-coherent and coherent transmission schemes, respectively. An interesting finding is that, for the coherent transmission scheme, the result of the identical signaling scheme for the MIMO double Rayleigh channel with independent branches is similar to that of the MIMO RFID channel under the
OSTBC scheme, except the roles of $N$ and $L$ are switched. The diversity order is given as $N$ regardless of $L$, but a significant BER performance improvement is observed from $L=1$ to $L=2$.

\section{Diversity comparison and performance bottleneck}

Under the identical signaling scheme, one interesting observation is that the diversity order of the studied MIMO RFID channel is $\min (N, L)$, as shown in Equation (24). Equation (23) implies that if $N-L>1$, solely increasing the number of receiving antennas $N$ does not enhance the BER performance significantly. Similarly, if $L-N>1$, solely increasing the number of tag antennas $L$ does not enhance the BER performance significantly either. Here, we refer to this observation as the performance bottleneck. In contrast, for both the Rayleigh channel with independent receiving branches and the double Rayleigh channel with independent receiving branches, the diversity order is $N$, which means solely increasing the number of receiving antennas can significantly enhance the BER performance. Also there is a significant BER improvement from $L=1$ to $L=2$ for the double Rayleigh channel with independent receiving branches, as shown in Equation (39). It is worth mentioning that, for the AF cooperative channel in Equation (17), the diversity order is $(L+N)$ [17].

Under the OSTBC scheme, the diversity order of the studied MIMO RFID backscattering channel is the number of tag antennas $L$, regardless of the number of the reader receiving antennas $N$, as shown in Equation (34). In contrast, for both the Rayleigh channel with independent receiving branches and the double Rayleigh channel with independent receiving branches, the diversity order is $N L$. The performance of the MIMO RFID channel is also significantly different from keyhole fading and double scattering fading. For the keyhole channel, the diversity order is $\min (N, L)$, and for the double scattering channel the diversity order is $M L N / \max (M, L, N)$.

Although the diversity order is always $L$ for the MIMO RFID channel with OSTBC, a significant BER improvement can still be observed from $N=1$ to $N=2$, because of the logarithm function in the numerator of the BER expression in Equation (33) when $N=1$. From 


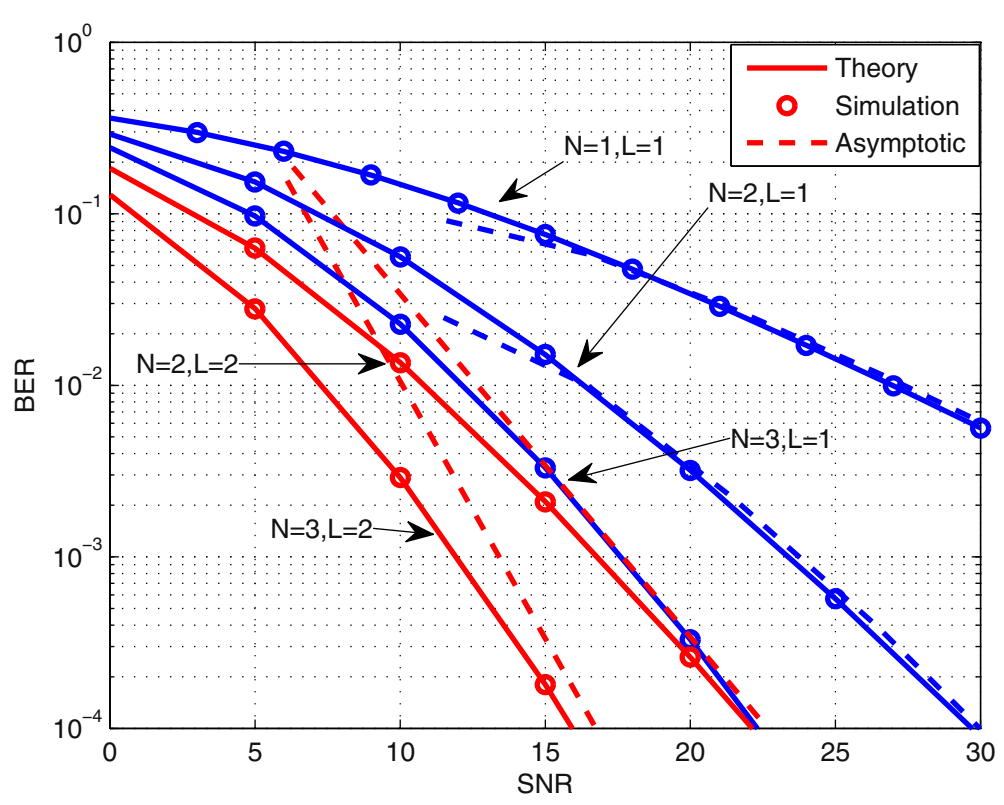

Figure 6 BER performances (in Equations 36 and 37) of the MIMO independent double Rayleigh channel using non-coherent identical signaling (FSK with EGC).

$N=2$ to $N>2$, the BER improvement is not significant, meaning that increasing the number of receiving antennas helps little when $N>2$. We consider this the performance bottleneck for the MIMO RFID backscattering channel under the OSTBC scheme. While for both Rayleigh channel with independent receiving branches and double Rayleigh channel with independent receiving branches, solely increasing the number of receiving or transmitting antennas can yield a higher diversity order and a significant BER performance improvement.

The above different behaviors of the MIMO RFID channel are due to the special structure of the MIMO RFID backscattering channel. All the above discussions are summarized in Table 3.

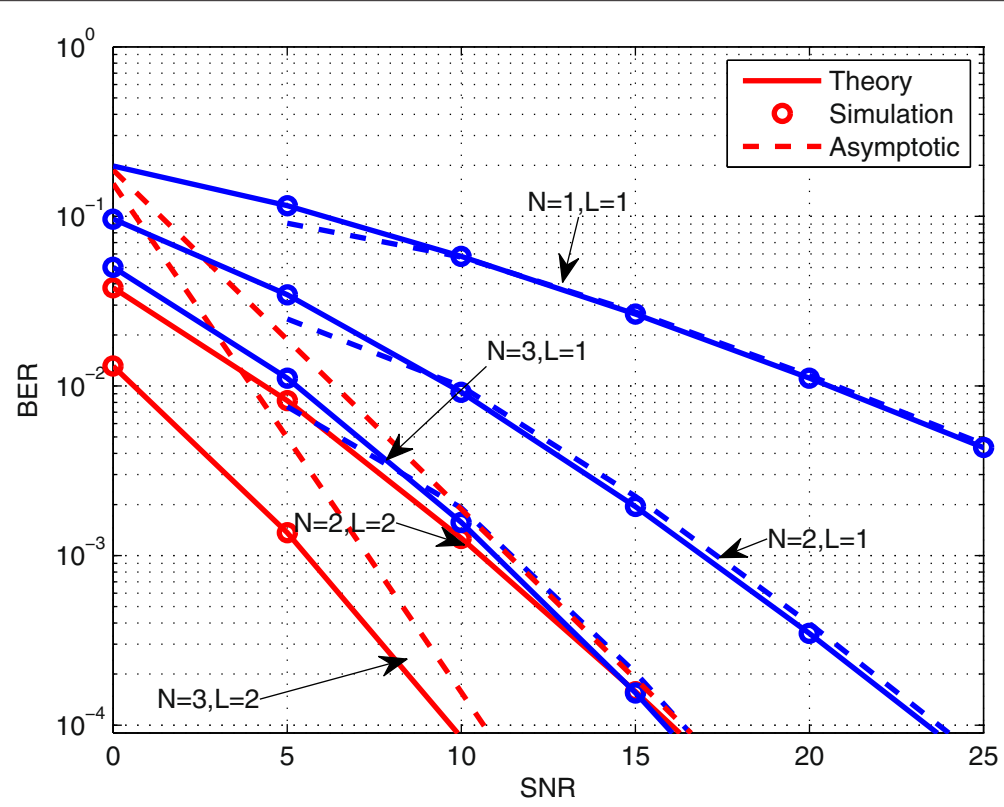

Figure 7 BER performances (in Equations 38 and 39) of the MIMO independent double Rayleigh channel using non-coherent identical signaling (BPSK with MRC) under perfect channel estimation. 


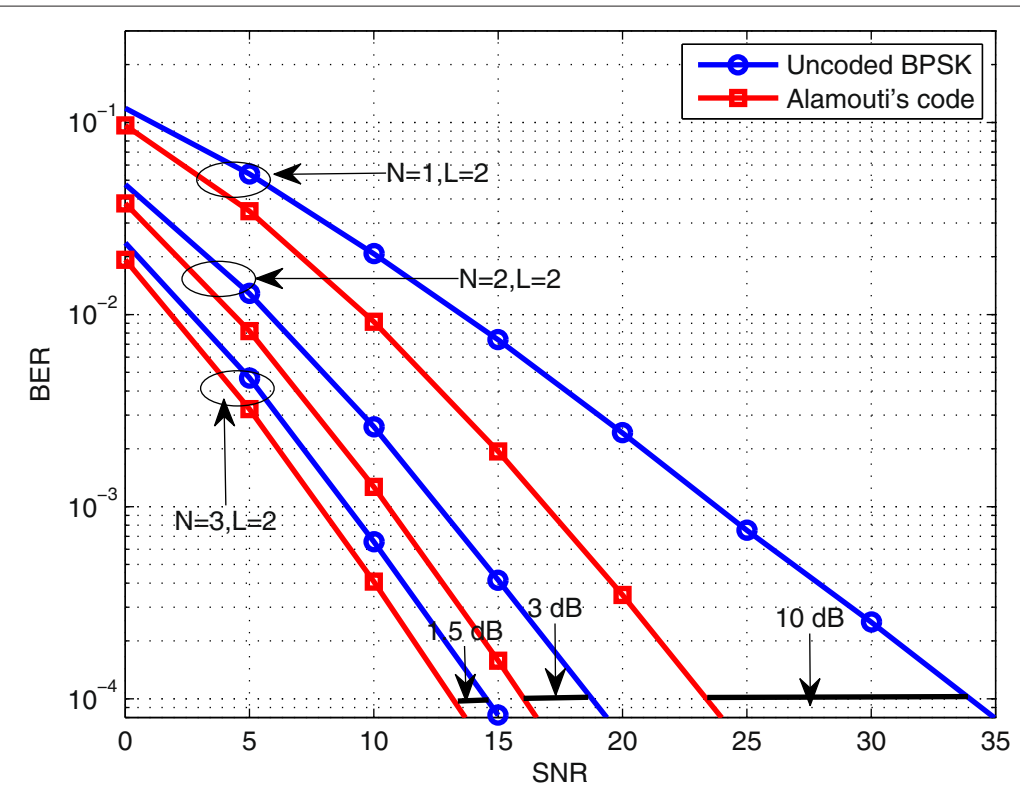

Figure 8 The BER performance comparison between Alamouti's coding scheme and identical signaling scheme. A significant BER improvement by Alamouti's code is observed for $N=1$, while the improvement is much smaller when $N \geq 2$. These properties can be explained by our analysis of the MIMO RFID channel under the OSTBC and identical signaling schemes.

Performance improvement by employing OSTBC in RFID backscattering channel

In this section, we investigate how much performance enhancement can be brought by employing OSTBC in the studied MIMO RFID channel. Figure 8 compares the BER performances of Alamouti's coding scheme and the identical signaling scheme in the $N \times L$ RFID channel, where the RF tag is equipped with 2 antennas (i.e., $L=2$ ) and the number of reader receiving antennas varies from 1 to 3. A significant performance improvement (about $10 \mathrm{~dB}$ ) is observed by Alamouti's coding scheme for the setting $N=1$. However, for the settings $N=2$ and $N=3$, the improvements by Alamouti's scheme are not significant (i.e., $3 \mathrm{~dB}$ for $N=2$ and $1.5 \mathrm{~dB}$ for $N=3$ ). This observation can be explained by the derived asymptotic BER expressions in Equations (24) and (34): Our analysis for the OSTBC scheme implies that for Alamouti's code the achievable diversity gain is $L$ ( $L=2$ in this example) for any $N$ in the $N \times L$ RFID channel. Consequently for the settings with $N \geq L$, Alamouti's code yields the same diversity order as that of the identical signaling scheme in the $N \times L$ RFID channel, and the BER performance improvement is limited. In other words, when $N \geq L$ in a MIMO RFID system, OSTBC does not yield significant performance improvement over simpler signaling schemes.

\section{Conclusion}

In this article, we analyzed the performance of the MIMO RFID backscattering channel, a cascaded channel that has a special kind of structure. We observe several interesting properties of the channel from our analytical results. First, the diversity order is $\min (N, L)$ when employing the identical signaling scheme and the diversity order is $L$ for the OSTBC signaling scheme. Second, the analytical results reveal that there is a performance bottleneck for the channel. More specifically, with the identical signaling scheme, when $|N-L|>1$ the BER performance enhancement is not significant by increasing the number of tag antennas or the number of receiving antennas alone. With the OSTBC signaling scheme, the SER performance enhancement is not significant when $N>L$. These properties of the MIMO RFID channel are significantly different from that of other types of cascaded channels such as keyhole and double scattering. The analytical results and observations presented in this article could provide a useful guidance for the design of RFID systems using multiple antennas.

\section{Appendix}

Proof of Proposition 1. Let $A=1+\overline{\bar{\gamma}} \sum_{l=2}^{L} \alpha_{l}$, then

$$
\begin{aligned}
& \int_{\alpha_{1}=0}^{\infty} \frac{\exp \left(-\alpha_{1}\right)}{A+\overline{\bar{\gamma}} \alpha_{1}} d \alpha_{1}=\int_{\alpha_{1}=0}^{\infty} \frac{\exp \left(-\alpha_{1}-\frac{A}{\overline{\bar{\gamma}}}\right)}{\overline{\bar{\gamma}}\left(\alpha_{1}+\frac{A}{\overline{\bar{\gamma}}}\right)} e^{\frac{A}{\overline{\bar{\gamma}}}} d\left(\alpha_{1}+\frac{A}{\overline{\bar{\gamma}}}\right) \\
& =\frac{e^{\frac{A}{\overline{\bar{\gamma}}}}}{\overline{\bar{\gamma}}} \int_{\alpha_{1}^{\prime}=\frac{A}{\overline{\bar{\gamma}}}}^{\infty} \frac{\exp \left(-\alpha_{1}^{\prime}\right)}{\alpha_{1}^{\prime}} d \alpha_{1}^{\prime}=\frac{e^{\frac{A}{\overline{\bar{\gamma}}}}}{\overline{\bar{\gamma}}} E_{1}\left(\frac{A}{\overline{\bar{\gamma}}}\right) .
\end{aligned}
$$


where $\alpha_{1}^{\prime}=\alpha_{1}+\frac{A}{\overline{\bar{\gamma}}}$ and $E_{1}(x)=\int_{t=x}^{\infty} e^{-t} / t d t$ are the special functions called the exponential integral [22]. Now we have

$$
\begin{aligned}
& \int_{\alpha_{2}=0}^{\infty} \cdots \int_{\alpha_{L}=0}^{\infty} \frac{e^{\frac{1}{\bar{\gamma}}}}{\overline{\bar{\gamma}}} E_{1}\left(\frac{1+\overline{\bar{\gamma}} \sum_{l=2}^{L} \alpha_{l}}{\overline{\bar{\gamma}}}\right) d \alpha_{L} \cdots d \alpha_{2} \\
& =\frac{e^{\frac{1}{\bar{\gamma}}}}{\overline{\bar{\gamma}}} \int_{\alpha_{2}=0}^{\infty} \ldots \int_{\alpha_{L}=0}^{\infty} \int_{t=1}^{\infty} \frac{\exp \left(\frac{t}{-\overline{\bar{\gamma}}}-t \sum_{l=2}^{L} \alpha_{l}\right)}{t} d t d \alpha_{L} \cdots d \alpha_{2} \\
& =\frac{e^{\frac{1}{\bar{\gamma}}}}{\overline{\bar{\gamma}}} \int_{t=1}^{\infty} \frac{e^{-\frac{t}{\gamma}}}{t}\left(\prod_{l=2}^{L} \int_{\alpha_{l}=0}^{\infty} \exp \left(-\alpha_{l} t\right) d \alpha_{l}\right) d t \\
& =\frac{e^{\frac{1}{\overline{\bar{\gamma}}}}}{\overline{\bar{\gamma}}} \int_{t=1}^{\infty} \frac{e^{-\frac{t}{\overline{\bar{\gamma}}}}}{t^{L}} d t=\frac{e^{\frac{1}{\overline{\bar{\gamma}}}}}{\overline{\bar{\gamma}}} E_{L}\left(\frac{1}{\overline{\bar{\gamma}}}\right) \doteq \begin{cases}\frac{\ln (\overline{\bar{\gamma}})}{\overline{\bar{\gamma}}}, & \text { if } L=1 ; \\
\frac{1}{(L-1) \overline{\bar{\gamma}}}, & \text { if } L>1 .\end{cases}
\end{aligned}
$$

The last step is obtained by the asymptotic property of the generalized exponential integral $E_{L}(\cdot)$ [22].

Proof of Proposition 2.

$$
\begin{aligned}
& \int_{\alpha=0}^{\infty}\left(\frac{1}{1+\overline{\bar{\gamma}} \alpha}\right)^{N} \exp (-\alpha) d \alpha=\int_{x=1}^{\infty} \frac{\exp \left(-\frac{x}{\bar{\gamma}}\right) \exp \left(\frac{1}{\gamma}\right)\left(\frac{1}{\bar{\gamma}^{N}}\right)}{\left(\frac{x}{\gamma}\right)^{N}} \frac{d x}{\overline{\bar{\gamma}}} \\
& =\int_{x^{\prime}=\frac{1}{\overline{\bar{\gamma}}}}^{\infty} \frac{1}{x^{\prime N}} \exp \left(-x^{\prime}\right) \exp \left(\frac{1}{\overline{\bar{\gamma}}}\right) \frac{1}{\overline{\bar{\gamma}} N} d x^{\prime}=\frac{E_{N}\left(\frac{1}{\overline{\bar{\gamma}}}\right)}{\overline{\bar{\gamma}}} \exp \left(\frac{1}{\overline{\bar{\gamma}}}\right)
\end{aligned}
$$

where $x=1+\overline{\bar{\gamma}} \alpha$, and $x^{\prime}=\frac{x}{\bar{\gamma}}$. The asymptotic expression is just like that in Proposition 2.

Prooffor Proposition 3. Let $A=1+\overline{\bar{\gamma}} \sum_{l=2}^{L} \alpha_{l}$ and

$$
\begin{aligned}
& \exp \left(-\sum_{l=2}^{L} \alpha_{l}\right) \int_{\alpha_{1}=0}^{\infty} \frac{1}{\left(A+\overline{\bar{\gamma}} \alpha_{1}\right)^{N}} \exp \left(-\alpha_{1}\right) d \alpha_{1} \\
& =\exp \left(-\sum_{l=2}^{L} \alpha_{l}\right) \frac{E_{N}\left(\frac{A}{\overline{\bar{\gamma}}}\right)}{A^{N-1 \overline{\bar{\gamma}}}} \exp \left(\frac{A}{\overline{\bar{\gamma}}}\right)=\exp \left(\frac{1}{\overline{\bar{\gamma}}}\right) \frac{E_{N}\left(\frac{A}{\overline{\bar{\gamma}}}\right)}{A^{N-1} \overline{\bar{\gamma}}},
\end{aligned}
$$

where $E_{N}(z)=\int_{t=1}^{\infty} \frac{e^{-z t}}{t^{N}} d t$ is the generalized exponential integral. Using the relation that $E_{N}(z)=\frac{1}{N-1}\left(e^{-z}-\right.$ $\left.z E_{N-1}(z)\right)[22]$ we can prove that

$$
\begin{aligned}
E_{N}(z)= & \frac{(-1)^{N-1} z^{N-1} E_{1}(z)}{(N-1) !} \\
& +\sum_{i=1}^{N-1} \frac{(-1)^{i-1}(N-1-i) ! z^{i-1} e^{-z}}{(N-1) !}
\end{aligned}
$$

then

$$
\begin{aligned}
& G_{N, L}(\overline{\bar{\gamma}})=\int_{\alpha_{L}=0}^{\infty} \cdots \int_{\alpha_{2}=0}^{\infty} e^{\frac{1}{\overline{\bar{\gamma}}}} \frac{E_{N}\left(\frac{A}{\overline{\bar{\gamma}}}\right)}{A^{N-1} \overline{\bar{\gamma}}} d \alpha_{2} \cdots \alpha_{L} \\
& =\int_{\alpha_{L}=0}^{\infty} \cdots \int_{\alpha_{2}=0}^{\infty} e^{\frac{1}{\overline{\bar{\gamma}}}} \frac{(-1)^{N-1}\left(\frac{A}{\overline{\bar{\gamma}}}\right)^{N-1} E_{1}\left(\frac{A}{\overline{\bar{\gamma}}}\right)}{A^{N-1} \overline{\bar{\gamma}}(N-1) !} \\
& +e^{\frac{1}{\bar{\gamma}}} \sum_{i=1}^{N-1} \frac{(-1)^{i-1}(N-1-i) !\left(\frac{A}{\overline{\bar{\gamma}}}\right)^{i-1} e^{-\frac{A}{\overline{\bar{\gamma}}}}}{(N-1) !} \\
& \times d \alpha_{2} \cdots d \alpha_{L}=\frac{e^{\frac{1}{\bar{\nu}}}}{\bar{\gamma}^{N}} \int_{\alpha_{L}}^{\infty} \cdots \int_{\alpha_{2}}^{\infty} \\
& \times \frac{(-1)^{N-1} E_{1}\left(\frac{A}{\bar{\gamma}}\right)}{(N-1) !} d \alpha_{2} \cdots d \alpha_{L}+\int_{\alpha_{L}}^{\infty} \cdots \int_{\alpha_{2}}^{\infty} \\
& \sum_{i=1}^{N-1} \frac{(-1)^{i-1}(N-1-i) ! e^{-\sum_{l=2}^{L} \alpha_{l}}}{(N-1) ! \overline{\bar{\gamma}}^{i}\left(1+\overline{\bar{\gamma}} \sum_{l=2}^{L} \alpha_{l}\right)^{N-i}} d \alpha_{2} \cdots d \alpha_{L} \\
& =\frac{1}{(-\overline{\bar{\gamma}})^{N-1}(N-1) !} G_{1, L}(\overline{\bar{\gamma}}) \\
& +\sum_{i=1}^{N-1} \frac{(-1)^{i-1}(N-1-i) !}{(N-1) ! \overline{\bar{\gamma}}^{i}} G_{(N-i)(L-1)}(\overline{\bar{\gamma}}) \\
& =\frac{1}{(-\overline{\bar{\gamma}})^{N-1}(N-1) !} G_{1, L}(\overline{\bar{\gamma}}) \\
& -\sum_{k=1}^{N-1} \frac{(k-1) !}{(-\overline{\bar{\gamma}})^{N-k}(N-1) !} G_{k(L-1)}(\overline{\bar{\gamma}}) .
\end{aligned}
$$

The last step is obtained by changing the index, i.e., $k=$ $N-i$.

Proof of Proposition 4. Case 1: $N>L$

We apply mathematical induction to prove this property. It is easy to show that $G_{N, 1}$ is valid for Equation (14), suppose for $L=j$ the argument is valid and our goal is to show for $L=j+1<N$ it is still valid. We have

$$
\begin{aligned}
G_{N,(j+1)}(\overline{\bar{\gamma}})= & \frac{1}{(-\overline{\bar{\gamma}})^{N-1}(N-1) !} G_{1(j+1)}(\overline{\bar{\gamma}}) \\
& -\sum_{k=1}^{N-1} \frac{(k-1) !}{(-\overline{\bar{\gamma}})^{N-k}(N-1) !} G_{k j}(\overline{\bar{\gamma}}) \\
\doteq & -\frac{1}{(-\overline{\bar{\gamma}})^{N}(N-1) !(j+1)} \\
& -\sum_{k=1}^{N-1} \frac{(k-1) !}{(-\overline{\bar{\gamma}})^{N-k}(N-1) !} G_{k j}(\overline{\bar{\gamma}})
\end{aligned}
$$




$$
\begin{aligned}
\doteq & -\frac{1}{(-\overline{\bar{\gamma}})^{N}(N-1) !(j+1)}-\frac{(N-2) !}{(-\overline{\bar{\gamma}})^{1}(N-1) !} \\
& \times G_{(N-1) j}(\overline{\bar{\gamma}}) \doteq-\frac{1}{(-\overline{\bar{\gamma}})^{N}(N-1) !(j+1)} \\
& +\frac{(N-2) !}{\overline{\bar{\gamma}}(N-1) !} \times \frac{1}{(N-2) \cdots(N-1-j) \overline{\bar{\gamma}}^{j}} \\
\doteq & -\frac{1}{(-\overline{\bar{\gamma}})^{N}(N-1) !(j+1)} \\
& +\frac{1}{(N-1) \cdots(N-1-j) \overline{\bar{\gamma}} j+1} \\
\doteq & \frac{1}{(N-1) \cdots(N-1-j) \overline{\bar{\gamma}}^{j+1}} .
\end{aligned}
$$

Therefore, Equation (14) is valid for $N>L$.

Case 2: $N=L$

For $N=1$ and $L=1$ it is easy to show Equation (14) is valid for $N>L$. Now we need to show that Equation (14) is still valid for $N=L=j+1$.

$$
\begin{aligned}
G_{(j+1),(j+1)}(\overline{\bar{\gamma}}) & \doteq \frac{1}{(-\overline{\bar{\gamma}})^{j} j !} G_{1(j+1)}(\overline{\bar{\gamma}})-\sum_{k=1}^{j} \frac{(k-1) !}{(-\overline{\bar{\gamma}})^{j+1-k j !}} G_{k j}(\overline{\bar{\gamma}}) \\
& \doteq-\frac{1}{(-\overline{\bar{\gamma}})^{j+1} j ! j}-\sum_{k=1}^{j} \frac{(k-1) !}{(-\overline{\bar{\gamma}})^{j+1-k_{j !}}} G_{k j}(\overline{\bar{\gamma}}) .
\end{aligned}
$$

Since for $k<j, G_{k j}(\overline{\bar{\gamma}}) \propto \frac{1}{\overline{\bar{\gamma}}^{k}}$ and for $k=j G_{k, j}(\overline{\bar{\gamma}}) \propto \frac{\ln (\overline{\bar{\gamma}})}{\overline{\bar{\gamma}}^{j}}$, we have

$$
\begin{aligned}
G_{(j+1),(j+1)}(\overline{\bar{\gamma}}) & \doteq-\frac{1}{(-\overline{\bar{\gamma}})^{j+1} j ! j}-\frac{(j-1) !}{(-\overline{\bar{\gamma}})^{j+1-j} j !} G_{j j}(\overline{\bar{\gamma}}) \\
& \doteq \frac{\ln (\overline{\bar{\gamma}})}{j ! \overline{\bar{\gamma}} j+1}
\end{aligned}
$$

Case 3: $N<L$

A similar approach as that of Case 1 can be obtained for this case; therefore, we omit the details here.

Proof for independent double Rayleigh. For the noncoherent transmission scheme, assuming that the instantaneous SNRs for $N$ independent receiving branches are $\gamma_{1}, \ldots, \gamma_{N}$, its BER expression with respective to the instantaneous SNRs can be expressed as

$$
P_{N, L}\left(\gamma_{t}\right)=\frac{1}{2^{2 N-1}} e^{-g \gamma_{t}} \sum_{k=0}^{N-1} b_{k}\left(g \gamma_{t}\right)^{k}
$$

where $\gamma_{t}=\sum_{n=1}^{N} \gamma_{n}$ and $b_{k}=\frac{1}{k !} \sum_{n=0}^{N-1-k}\left(\begin{array}{l}2 N-1 \\ n\end{array}\right)$. Note that $\gamma_{t}^{k}=\left(\gamma_{1}+\cdots+\gamma_{N}\right)^{k}=\sum_{\mathbf{m} \in \mathbb{M}_{k}}\left(\begin{array}{l}k \\ \mathbf{m}\end{array}\right) \gamma_{1}^{m_{1}} \cdots \gamma_{1}^{m_{N}}$, where $\mathbf{m} \triangleq\left[m_{1}, \ldots, m_{N}\right]$, and $\mathbb{M}_{k} \triangleq\left\{\mathbf{m} \mid m_{1}+\cdots+m_{N}=\right.$ $k\}$; therefore, we have

$$
P_{N, L}\left(\gamma_{t}\right)=\frac{1}{2^{2 N-1}} \sum_{k=0}^{N-1} b_{k} g^{k} \sum_{\mathbf{m} \in \mathbb{M}_{k}}\left(\begin{array}{l}
k \\
\mathbf{m}
\end{array}\right) \prod_{n=1}^{N} e^{-g \gamma_{n}} \gamma_{n}^{m_{n}}
$$

Averaging the densities of $\gamma_{n}$ according to Equation (8), we have the BER expression for the non-coherent transmission scheme as

$$
\begin{aligned}
P_{N, L}(\bar{\gamma})= & \frac{1}{2^{2 N-1}} \sum_{k=0}^{N-1} b_{k} g^{k} \sum_{\mathbf{m} \in \mathbb{M}_{k}}\left(\begin{array}{l}
k \\
\mathbf{m}
\end{array}\right) \prod_{n=1}^{N} \int_{\gamma_{n}=0}^{\infty} \\
& \times e^{-g \gamma_{n}} \gamma_{n}^{m_{n}} f_{\gamma_{n}}\left(\gamma_{n}\right) d \gamma_{n} \\
= & \frac{1}{2^{2 N-1}} \sum_{k=0}^{N-1} b_{k} \sum_{\mathbf{m} \in \mathbb{M}_{k}}\left(\begin{array}{l}
k \\
\mathbf{m}
\end{array}\right) \prod_{n=1}^{N} \frac{\Gamma\left(L+m_{n}\right) \Gamma\left(1+m_{n}\right)}{\Gamma(L) g \bar{\gamma}} \\
& \times U\left(L+m_{n}, L ; \frac{1}{g \bar{\gamma}}\right),
\end{aligned}
$$

where evaluation of the integral can be found in [22] and $U(a, b, x)$ is the confluent hypergeometric function. For small values of $x$, the asymptotic expressions of $U(a, b, x)$ are given by [19]

$$
U(a, b, x) \doteq \begin{cases}-\frac{1}{\Gamma(a)} \ln (x), & \text { if } b=1 \\ \frac{\Gamma(b-1)}{\Gamma(a)} x^{1-b} & \text { if } b>1\end{cases}
$$

Therefore, for large value of $\bar{\gamma}$, we can derive the following asymptotic BER expression

$P_{N, L}(\bar{\gamma}) \doteq \begin{cases}\frac{1}{2^{2 N-1}} \sum_{k=0}^{N-1} b_{k} \sum_{\mathbf{m} \in \mathbb{M}_{k}}\left(\begin{array}{l}k \\ \mathbf{m}\end{array}\right) \prod_{n=1}^{N} \frac{\Gamma(m+1)}{\Gamma(L)} \frac{\ln (g \bar{\gamma})}{g \bar{\gamma}}, & \text { if } L=1 ; \\ \frac{1}{2^{2 N-1} \sum_{k=0}^{N-1} b_{k} \sum_{\mathbf{m} \in \mathbb{M}_{k}}\left(\begin{array}{ll}k \\ \mathbf{m}\end{array}\right) \prod_{n=1}^{N} \frac{\Gamma(m+1)}{(L-1) g \bar{\gamma}},} & \text { if } L>1 .\end{cases}$

For the coherent transmission scheme, we apply the method introduced in [20]. For $N$ independent receiving branches, the BER for coherent transmission can be expressed as

$$
P_{N, L}(\bar{\gamma})=\frac{1}{\pi} \int_{\theta=0}^{\pi / 2} I(\theta, \bar{\gamma})^{N} d \theta
$$

Here $I(\theta, \bar{\gamma})=\int_{\gamma=0}^{\infty} f_{\gamma}(\gamma) \exp \left(-\frac{g \bar{\gamma} \gamma}{\sin (\theta)}\right) d \gamma$, where $\gamma=$ $\sum_{n=1}^{N} \gamma_{n}$ and $f_{\gamma}(\cdot)$ means its density function. With $\overline{\bar{\gamma}}=$ $\frac{g \bar{\gamma}}{\sin \theta}$, we have $I(\theta, \bar{\gamma})=G_{1, L}(\overline{\bar{\gamma}})$. The BER for the identical 
signaling MIMO setting of $L$ transmitting antennas and $N$ receiving antennas is expressed as

$$
\begin{aligned}
& P_{N, L}(\bar{\gamma})= \frac{1}{\pi} \int_{\theta=0}^{\pi / 2}\left(G_{1, L}(\overline{\bar{\gamma}})\right)^{N} d \theta=\frac{1}{\pi} \int_{\theta=0}^{\pi / 2} \\
& \times\left(\frac{e^{\frac{1}{\bar{\gamma}}}}{\overline{\bar{\gamma}}} E_{L}\left(\frac{1}{\overline{\bar{\gamma}}}\right)\right)^{N} d \theta \\
& \doteq \begin{cases}\frac{1}{\pi} \int_{\theta=0}^{\pi / 2}\left(\frac{\ln (\overline{\bar{\gamma}})}{\overline{\bar{\gamma}}}\right)^{N} d \theta=C_{N}\left(\frac{\ln (\bar{\gamma})}{\bar{\gamma}}\right)^{N}, \quad \text { if } L=1 ; \\
\frac{1}{\pi} \int_{\theta=0}^{\pi / 2}\left(\frac{1}{(L-1) \overline{\bar{\gamma}}}\right)^{N} d \theta=C_{N}\left(\frac{1}{(L-1) \bar{\gamma}}\right)^{N}, \quad \text { if } L>1 .\end{cases}
\end{aligned}
$$

\section{Competing interests}

The authors declare that they have no competing interests.

\section{Acknowledgements}

This study was supported by the Natural Sciences and Engineering Research Council of Canada (NSERC) under STPGP 364962-08.

\section{Author details}

${ }^{1}$ Department of Electrical and Computer Engineering, University of British Columbia, Vancouver, Canada. ${ }^{2}$ Department of Electrical Engineering, State University of New York (SUNY) at Buffalo, Buffalo, NY, USA.

Received: 25 August 2012 Accepted: 7 November 2012 Published: 29 November 2012

\section{References}

1. R Want, An introduction to RFID technology. IEEE Pervasive Comput. 13, 25-33 (2006)

2. F Klaus, RFID Handbook: Fundamentals and Applications in Contactless Smart Cards and Identification, 2nd edn. (Wiley, Hoboken, NJ, 2003)

3. P Nikitin, KVS Rao, in IEEE Antennas and Propagation Society International Symposium. Performance limitations of passive UHF RFID systems, (9-14 July 2006), pp. 1011-1014

4. GD Vita, G lannaccone, Design criteria for the RF section of UHF and microwave passive RFID transponders. IEEE Trans. Microw. Theory Tech. 53, 2978-2990 (2005)

5. D Yeager, F Zhang, A Zarrasvand, BP Otis, in ISSCC 2010. A 9.2 uA gen 2 compatible UHF RFID sensing tag with $-12 \mathrm{dBm}$ sensitivity and 1.25 uVrms input-referred noise floor, San Francisco, USA, 7-11 Feb 2010), pp. 1011-1014

6. PV Nikitin, KS Rao, in IEEE International Symposium on Antennas and Propagation. Gain measurement of antennas using RFID, (3-8 July 2011), pp. 1012-1015

7. JD Griffin, GD Durgin, Multipath fading measurements for multi-antenna backscatter RFID at $5.8 \mathrm{GHz}$. IEEE Trans. Antennas Propagat. 58, 3693-3700 (2010)

8. D Kim, M Ingram, W Smith, Measurements of small-scale fading and path loss for long range RF tags. IEEE Trans. Antennas Propagat. 51, 1740-1749 (2003)

9. JD Griffin, GD Durgin, Gains for RF tags using multiple antennas. IEEE Trans. Antennas Propagat. 56, 563-570 (2008)

10. JD Griffin, GD Durgin, Gains For RF tags using multiple antennas. IEEE Commun. Lett. 11, 735-737 (2007)

11. M Ingram, M Demirkol, D Kim, in International Symposium on Signals, Systems, and Electronics. Transmit diversity and spatial multiplexing for RF links using modulated backscatter, Tokyo, Japan, 24-27 Jul 2001), pp. 1-4

12. S Sarkka, M Huusko, W Viikari, K Jaakkola, Phase-based UHF RFID tracking with nonlinear Kalman filtering and smoothing. IEEE Sensors J. 12, 904-910 (2012)

13. JD Griffin, GD Durgin, Complete link budgets for backscatter radio and RFID systems. IEEE Antennas Propagat. Mag. 51, 11-25 (2009)
14. B Strassner, K Chang, Passive 5.8-GHz radio-frequency identification tag for monitoring oil drill pipe. IEEE Trans. Microwave Theory Tech. 51, 356-363 (2003)

15. H Shin, JH Lee, Effect of keyholes on the symbol error rate of space-time block codes. IEEE Commun. Lett. 7, 27-29 (2003)

16. Y Gong, KB Letaief, On the error probability of orthogonal space-timeblock codes over keyhole MIMO channel. IEEE Trans. Wirel. Commun. 6, 2402-2409 (2007)

17. M Elkashlan, PL Yeoh, RHY Louie, I Collings, On the exact and asymptotic SER of receive diversity with multiple amplify-and-forward relays. IEEE Trans. Veh. Technol. 59, 4602-4608 (2011)

18. H Shin, MZ Win, MIMO diversity in the presence of double scattering. IEEE Trans. Inf. Theory. 54, 2976-2996 (2008)

19. M Abramowitz, IA Stegun, Handbook of Mathematical Functions, 10th edn. (National Bureau of Standards, Washington, DC, 1972)

20. M Simon, M Alouini, A unified approach to the performance analysis of digital communication over generalized fading channels. Proc. IEEE. 86, 1860-1877 (1998)

21. DG Brennanr, Linear diversity combining techniques. Proc. IEEE. 91, 331-356 (2003)

22. I Gradsheteyn, I Ryzhik, Table of Integrals, Series and Product, 7th edn. (Academic Press, New York, 1994)

23. J Proakis, Digital Communications, 4th edn. (McGraw-Hill, New York, 2001)

24. C Zhu, J Mietzner, R Schober, On the performance of non-coherent transmission schemes with equal-gain combining in generalized K-fading. IEEE Trans. Wirel. Commun. 9, 1337-1349 (2010)

25. C He, ZJ Wang, Closed-form BER analysis of non-coherent FSK in MISO double rayleigh fading/RFID channel. IEEE Commun. Lett. 15, 848-850 (2011)

26. SM Alamouti, A simple transmit diversity technique for wireless communications. IEEE J. Sel. Areas Commun. 16, 1451-1458 (1998)

27. V Tarokh, H Jafarkhani, A Calderbank, Space-time block coding for wireless communications: performance results. IEEE J. Sel. Areas Commun. 17, 451-460 (1999)

28. S Sandhu, A Paulraj, Space-time block codes: a capacity perspective. IEEE Commun. Lett. 10, 384-386 (2000)

29. V Tarokh, N Seshadri, A Calderbank, Space-time codes for high data rate wireless communication: performance criterion and code construction. IEEE Trans. Inf. Theory. 44, 744-765 (1998)

30. M Uysal, Diversity analysis of space-time coding in cascaded Rayleigh fading channels. IEEE Commun. Lett. 10, 165-167 (2006)

\section{doi:10.1186/1687-1499-2012-357}

Cite this article as: He et al.: On the performance of MIMO RFID backscattering channels. EURASIP Journal on Wireless Communications and Networking 2012 2012:357.

\section{Submit your manuscript to a SpringerOpen ${ }^{\circ}$ journal and benefit from:}

- Convenient online submission

Rigorous peer review

- Immediate publication on acceptance

- Open access: articles freely available online

- High visibility within the field

- Retaining the copyright to your article

Submit your next manuscript at $>$ springeropen.com 